\title{
Performance Evaluation of Input Devices in Virtual Environments
}

\author{
Andreas Roessler, Volker Grantz \\ Fraunhofer IAO \\ Nobelstrasse 12, 70569 Stuttgart, Germany, \\ Andreas.Roessler@iao.fhg.de
}

\begin{abstract}
The user interface approach of virtual reality promises to be superior to twodimensional approaches. Therefore, there is a need to perform experiments with different input devices. We developed a virtual environment test bed which integrates different input devices and modules for rapid modelling tests and evaluation. Our focus of the tests was a comparison between a conventional computer mouse, a space mouse and an electromagnetically tracked device. With the tests, we tried to measure the accuracy and performance of grabbing and positioning virtual objects.
\end{abstract}

\section{Keywords}

usability, evaluation, virtual reality, input devices, $3 \mathrm{~d}$ tracking

\section{INTRODUCTION}

Until today there are very few highly interactive applications of virtual reality technology. Most of the applications around the globe focus on the visualisation of information - the used interaction is restricted to simple walk-throughs. As it happens quite often even the design of the "simple“ walk-through-interaction is too difficult for normal users.

VR-evangelists even dream of highly interactive applications where the immersed user is able to interact with any kind virtual object very intuitively. To meet this goal, we need to understand the characteristics of virtual environments, three-dimensional input devices and the basic tasks that have to be performed.

Like other VR groups, who develop various interactive applications for industrial partners and for research purposes, we urgently need this kind of better understanding of man-machine-interactions in virtual environments.

In a first series we tested the ergononmic issues of VR systems /Deisinger and Riedel 1996/ and user interactions in a CAVE-like projection environment /Blach, Simon and Riedel 1997/. The aim of the tests described in this paper is the evaluation of different input devices and an analysis of their characteristics. Design of Experiments 


\subsection{Aspects of Evaluation}

The objective of our experiments was to identify characteristics of different input devices. The task to be fulfilled were grabbing and accurate positioning of virtual objects. We were especially interested in the following aspects:

- Efficiency

How fast can users move virtual objects to reference positions?

- Accuracy

How accurate do the final positions match the objects with the references?

- Users' Satisfaction

What are the users subjective opinions concerning the input devices?

\subsection{The Input Devices and their Interaction Modes}

As evaluation devices for our test we selected the standard computer mouse, the spacemouse and a simple self-designed device, consisting of a sensor of an electromagnetic tracking system and a button. We did not include an dataglove because of the following reasons: First, the test does not focus on grabbing the objects but on their positioning. Second, there are ergonomic reasons which exclude the datagloves from broader use: all available datagloves have only one size - at least in our lab. Therefore, the gloves do not fit for very small and very big hands, and need to be calibrated by the software. Additionally, taking on and off the gloves is inconvenient for the users.

\section{The Computer Mouse}

We included the mouse (see figure 1a) in our test because there are a lot of users who are familiar with it as well as many CAD and modelling packages which use the mouse as a three-dimensional input device. The typical problem is how to map all six degrees of freedom to a two-dimensional device. For the test, we mapped the movements as shown in the following table:

Table 1 Mouse mappings

\begin{tabular}{ll}
\hline Pressed button $(s)$ & Movements \\
\hline Left Button & Forward \& backward, left \& right \\
Right Button & Up \& down, left \& right \\
Left \& Right Button & Heading + \& -, pitch $+\&-$ \\
Middle & Grab an object, if collided \\
\hline
\end{tabular}

The Spacemouse

The spacemouse (see figure $1 \mathrm{~b}$ ) is a commercially available threedimensional input device which is frequently used for $\mathrm{CAD}$. With a spacemouse, the users are able to define the speed of movement and rotation of virtual objects by pressing a slightly movable half sphere. The half sphere can be pushed left, right, forward, backward, up and down, and the grabbed virtual objects move accordingly. By applying a torque to the half sphere, the virtual objects rotate. The speed of rotation and the position movements of the objects are set proportionally to the applied force and torque. 
The spacemouse offers six programmable buttons; we used button 1 for grabbing virtual objects and button 3 to reset the orientation. Additionally the spacemouse includes special features by hardware, e.g. an input mode which disables all degrees of freedom but the one with the highest input values. As the other tested input devices do not include that kind of assistance, we did not use these modes for our tests.

\section{The Tracked Button}

The tracked button (see figure 1c and 1d) is a simple device designed by the author, consisting of a sensor of the electromagnetic tracking system and a button. The button is polled by a micro-controller, which is connected via RS232 with the host computer. The purpose of the button is to grab virtual objects. The tracking system provides absolute values for position and orientation relative to a physical reference point which is set by the electromagnetic transmitter. A logical reference point was set in a comfortable initial position defined by the subject.
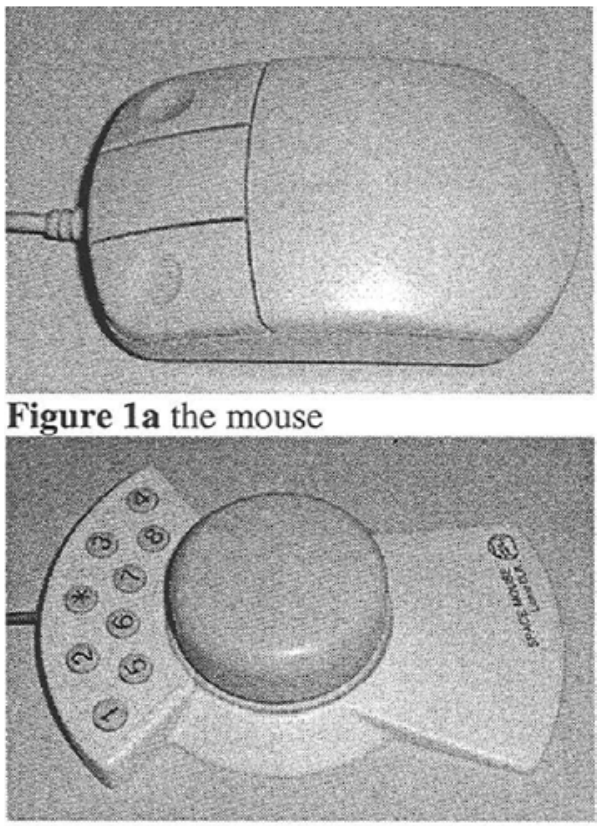

Figure $1 \mathbf{b}$ the spacemouse

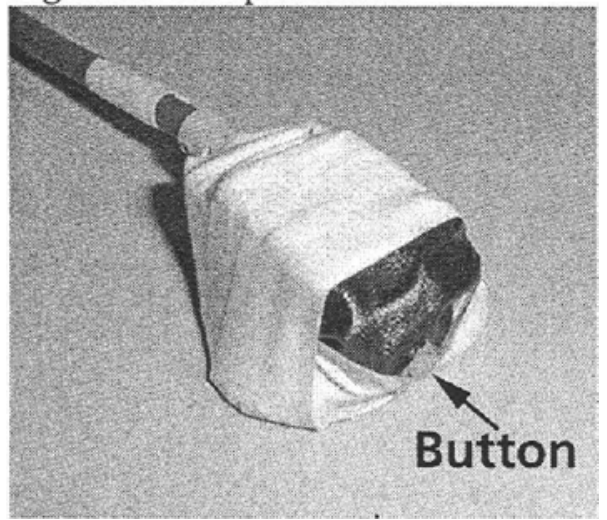

Figure 1c the tracked button

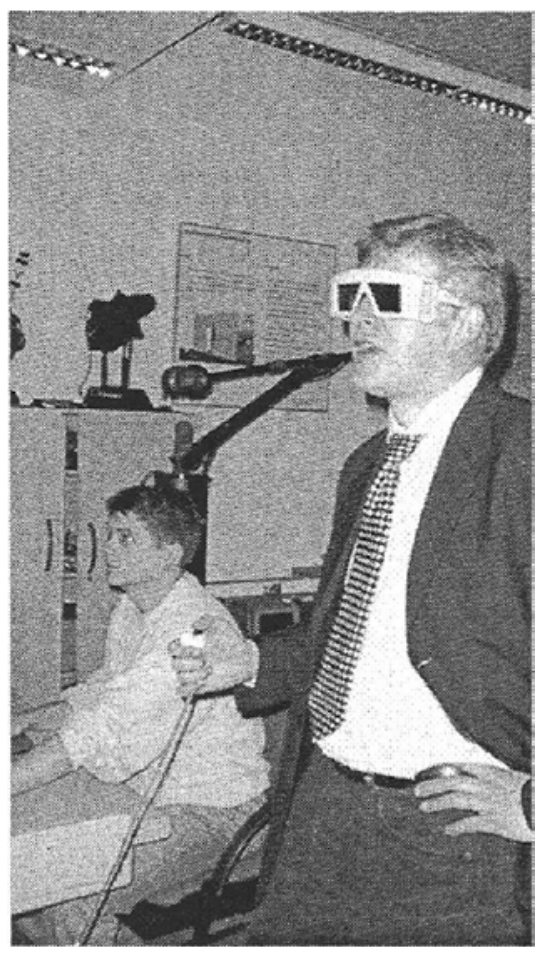

Figure 1d a subject with the tracked button during the test 


\subsection{Hardware \& Software}

The tests were developed with the VR-kernel Lightning /Blach et al 1998/, a development of Fraunhofer IAO. It consists of a rendering engine based on SGI Performer, device drivers for input and output devices, a routing manager that controls the application and a $\mathrm{C} / \mathrm{C}++$ application programming interface. Additionally, it includes a high-level scripting language, which is an extension of TCL (Tool Command Language /Ousterhout/). The complete test suite including the definition of the input and output devices, the interaction modes and the protocol recording was realised in TCL. For the control of the test suite by the supervisor, a GUI was built with TK, another extension of TCL. The GUI and Lightning communicated via TCP/IP. As a hardware platform we used a SGI Onyx with two RealityEngines2 and six processors. The tracking device was a Motionstar Extended Range from Ascension.

\subsection{The Test Environment \& Realisation}

The virtual environment (see figure 2) was presented to the user wearing shutterglasses on a large screen $(3 \mathrm{~m} \times 2 \mathrm{~m})$ stereoscopic video projection (Barco, resolution 960x960, frequency $96 \mathrm{hz}$ ). The virtual viewpoint was not tracked but was fixed by the test supervisor. The subjects sat (with mouse or spacemouse) or stood (with the tracked button) in front of the screen.

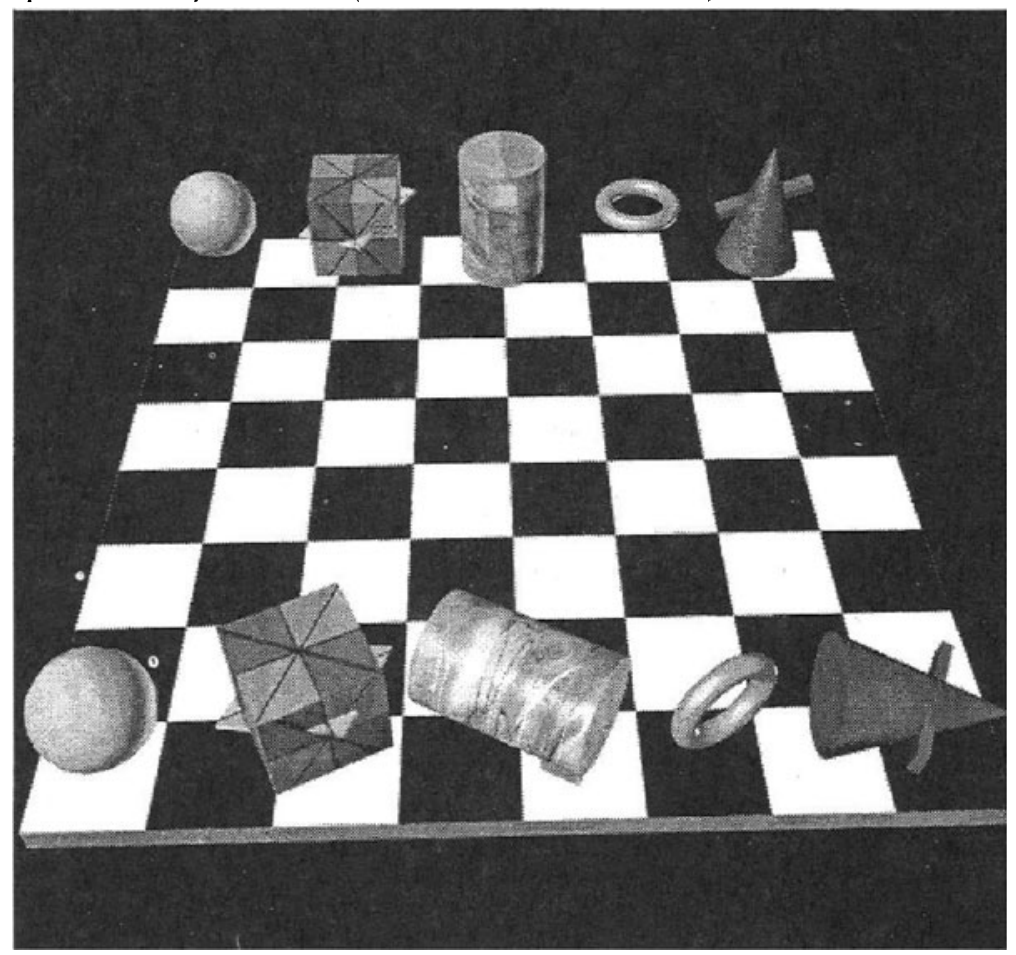

Figure 2 the virtual test environment

Before the test, the subjects had to fill in a questionnaire, that helped us to check their specific experience. After the test, we asked them to name the preferred input device.

The test included four similar tasks: the subject had to grab one of the four objects from the foreground and to move it to the reference position in the background. The first object was a sphere where rotation was not considered. The other objects had to be positioned and oriented to cover the reference objects perfectly. 
The subjects had to move the object with each of the considered input devices. Before the tests, the supervisor explained the devices and the users had the opportunity to get acquainted with the input devices and with the grabbing of the virtual objects. All subjects performed the tests in the same order: mouse, spacemouse and last the tracked button.

The used interaction technique was the same in the three tests: the input device directly controlled a three-dimensional cursor, represented by virtual tongs. An object could be grabbed when the cursor collided with it. The collision and the successful grabbing was visualised by a change of colour and shape of the virtual tongs.

In total, twenty subjects performed the tests. All of them had used the computer mouse for mainly two-dimensional applications before, none of them had experience with the spacemouse and three subjects were familiar with the use of a tracked button.

All movements of the cursor and all grab actions were recorded in a protocol file for evaluation purposes. From the files, the resulting period and accuracy was calculated automatically.

Similar tests had been performed by Hinckley et. al. /1997/, who compared pure rotation of virtual objects with mouse and tracked devices. Poupyrev et. al. /1997/ developed a framework for the evaluation of immersive direct manipulation, but did not focus on the comparison of devices.

\section{RESULTS}

The following results were received from an analysis of the protocol files recorded during our test sessions. We tried to obtain information on efficiency and accuracy from the files and subjective judgement of the users.

\subsection{Measured Values}

Figure 3 shows the average time the users needed to complete the tests. It shows, that the use of the tracked button is significantly faster than the two other devices.

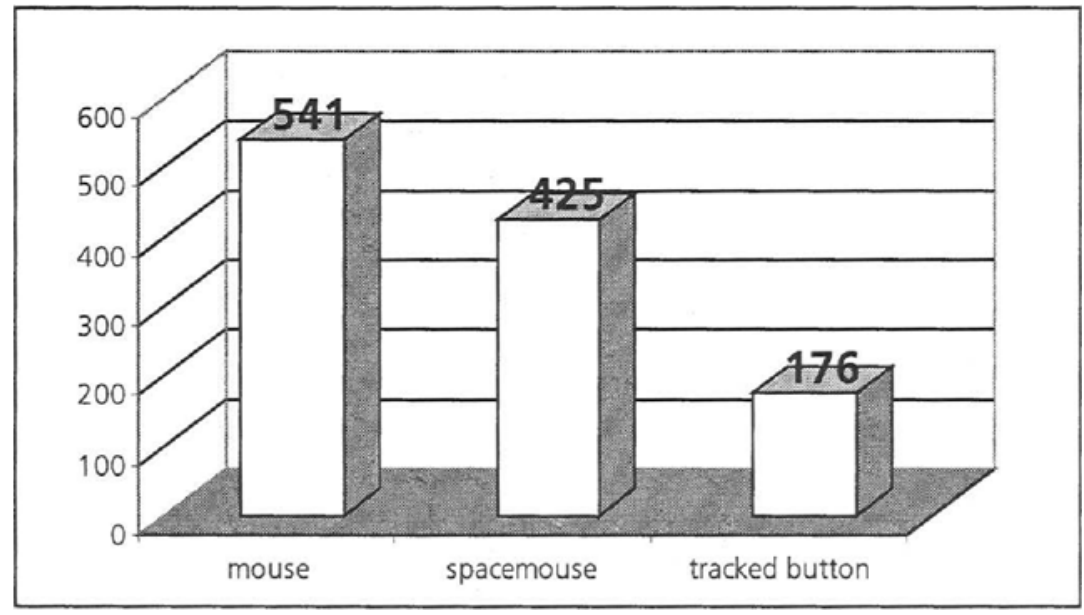

Figure 3 average total time in seconds 


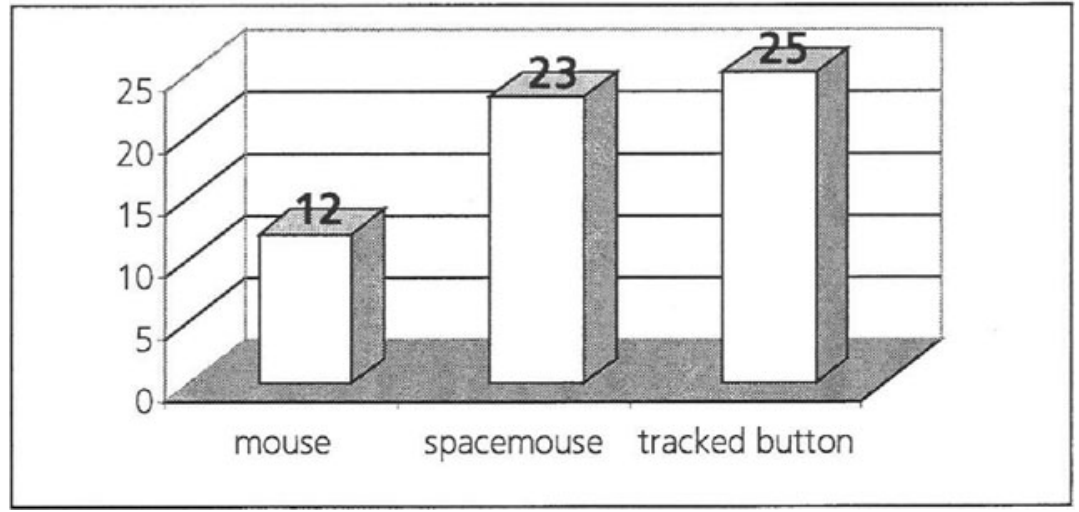

Figure 4 average time in seconds to move object 1 (sphere)

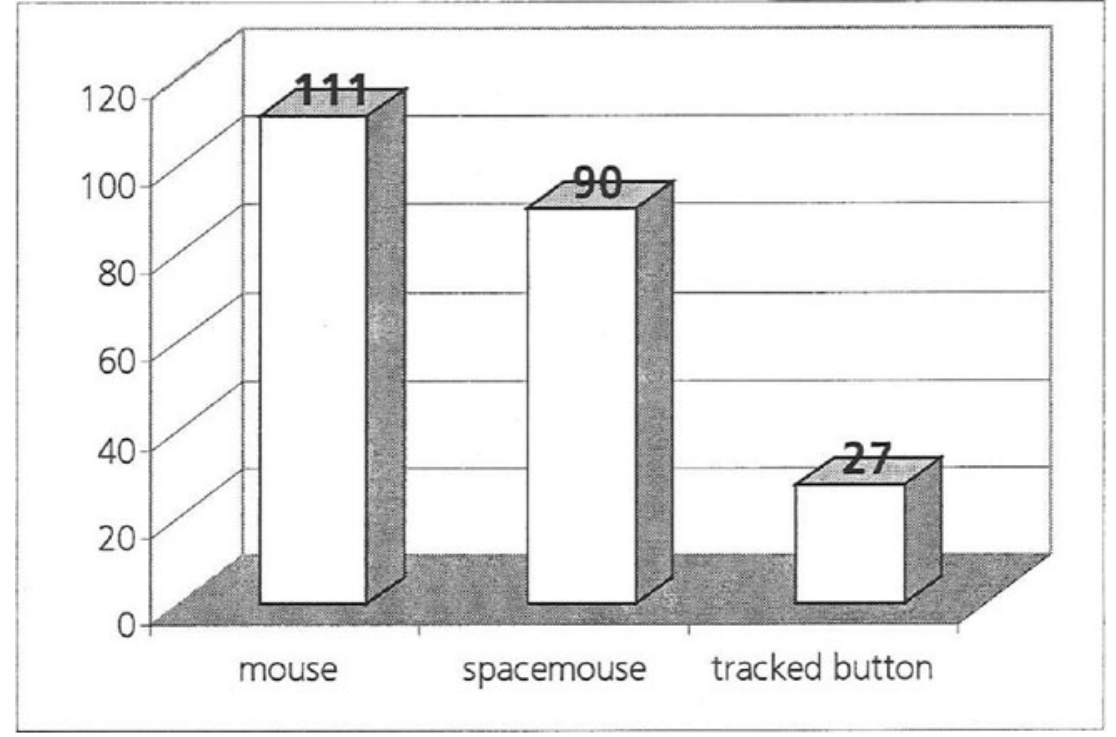

Figure 5 average time in seconds to move object 5: (cone)

Figures 4 and 5 compare the positioning of object 1 , which did not need rotation, and object 5 . It is interesting, that the interaction with the tracked button took approximately the same time even when the rotation was needed. The time difference between tracker button compared with mouse and spacemouse results mainly from the difficulties in orientating the objects correctly. The results fit to Hinckley's /1997/ who concluded that rotation of virtual objects with tracked devices is $36 \%$ faster than with the mouse.

Table 2 average deviation and range for positioning of object 3 (cylinder), $\mathrm{X}-, \mathrm{Y}-, \mathrm{Z}$-axes

\begin{tabular}{lrrr}
\hline & Mouse & Spacemouse & Tracked button \\
\hline Average deviation X & 0,249 & 0,394 & 0,42 \\
Range of deviation X & 0,112 & 0,089 & 0,165 \\
& 0,386 & 0,699 & 0,675 \\
Average deviation Y & 0,997 & 0,76 & 1,028 \\
Range of deviation Y & 0,062 & 0,277 & 0,52 \\
& 1,932 & 1,243 & 1,536
\end{tabular}




\begin{tabular}{lrrr} 
Average deviation Z & 1,02 & 0,872 & 0,744 \\
Range of deviation Z & 0,209 & 0,329 & 0,288 \\
& 1,831 & 1,415 & 1,2 \\
\hline
\end{tabular}

The results of the positioning (see table 2) show, that sufficient accuracy (for the subjects) was reached in similar ranges by all input devices.

Table 3 shows the deviation range for the orientation of two objects. The tracked button has the lowest absolute deviation and the lowest variation between different users.

Table 3 average deviation range for orientation of object 3 (cylinder) and 5 (cone)

\begin{tabular}{lrrrrrr}
\hline & & Object & 3 & & Object & $\begin{array}{r}5 \\
\text { Heading }\end{array}$ \\
& Pitch & Roll & Heading & Pitch & Roll \\
\hline Mouse & 0,699 & 1,771 & 0,303 & 3,781 & 2,313 & 1,5 \\
& 11,568 & 9,457 & 6,402 & 18,805 & 12,735 & 8,902 \\
Space- & 1,119 & 1,465 & 0,355 & 2,313 & 2,64 & 0,043 \\
mouse & 7,729 & 6,587 & 2,617 & 19,789 & 13,776 & 9,387 \\
Tracked & 1,194 & 0,588 & 0,549 & 0,631 & 0,657 & 0,613 \\
button & 5,93 & 5,584 & 3,259 & 11,927 & 7,121 & 5,991 \\
\hline
\end{tabular}

\subsection{Assessment of the Users}

After the test all users filled in a questionnaire in order to assess their subjective opinion on the different input devices. 85 percent (17 users) preferred the tracked button, 10 percent (2) the spacemouse and one user (5 $\%)$ preferred the standard mouse.

The following table shows the answers of the subjects on specific usability criteria:

Table 4 Average subjective assessment of the users (values range $1-6$, best:

1)

\begin{tabular}{llll}
\hline & Mouse & Spacemouse & Tracker \\
\hline Accuracy & 2,632 & 2,842 & 3,052 \\
Efficiency & 4,263 & 3,421 & 1,368 \\
Overall usability & 3,579 & 3,158 & 1,737 \\
Learnability & 3,368 & 3,105 & 1,368 \\
\hline
\end{tabular}

Again, the users ranked the tracked button as number one in the criteria efficiency, overall usability and learnability. They were not happy with the accuracy of the tracked button - an answer which does not match with the measured values. 


\section{CONCLUSIONS}

We know from discussions with experienced spacemouse users, that this input device can be very efficient and accurate. Nevertheless our tests show, that new users definitely prefer the tracked button which seems to be much more intuitive and efficient. The reason is the direct mapping between the orientation of the tracked button and the virtual object. The assessment by the (inexperienced) subjects shows, that they dislike especially three-dimensional rotation with mouse and spacemouse in combination with the used mappings. A paradox conclusion is that the tracked button is both accurate, shown by the measurements, and inaccurate, perceived by the subjects.

Therefore, our research focus will be the improvement of the accuracy and the ergonomics of the use of tracked devices. Additionally, we will consider an aspect that has not been included in our recent tests: In order to grab objects which are not within reach we need to implement and test additional features for the use of a tracked button.

\section{LITERATURE}

Blach, R.; Simon, A.; Rösch, A.; Landauer, J.( 1998) A Highly Flexible Virtual Reality System. In: Future Generation Computer Systems. Elsevier Science. In print.

Blach, R.; Simon, A.; Riedel, O. (1997) Experiences with User Interactions in a CAVE-like Projection Environement. In: Smitz, M.J.; Salvendy, G.; Koubek, R.J. (Eds.): Advances in Human Factors/Ergonomics. Elsevier Science.

Deisinger, J.; Riedel, O. (1996) Ergonomic Issues of Virtual Reality Systems: Head Mounted Displays. In: Virtual Reality World 1996. Conference documentation. Computerwoche Verlag, München.

Hinckley, K., Tullio, J., Pausch, R., Proffitt, D., Kassell, N. (1997) Usability Analysis of 3D Rotation Techniques, Proc. ACM UIST'97 Symposium on User Interface Software \& Technology, pp. 1-10.

Ousterhout, J. K. (1994) TCL and the TK Toolkit. Addison-Wesley.

Poupyrev, I.; Weghorst, S.; Billinghurst, M.; Ichikawa, T. (1997) A Framework and Testbed for Studying Manipulation Techniques for Immersive VR: VRST 97. ACM. 